EPJ Web of Conferences 16, 06006 (2011)

DOI: $10.1051 /$ epjconf/20111606006

(C) Owned by the authors, published by EDP Sciences, 2011

\title{
TNO, occultation, and high angular resolution astronomy
}

\author{
S.-K. King ${ }^{a}$ \\ Institute of Astronomy and Astrophysics, Academia Sinica, PO Box 23-141, Taipei 106, \\ Taiwan, R.O.C.
}

\begin{abstract}
The trans-Neptunian region is a new frontier in the solar system research. Occultation is currently the only way to detect a trans-Neptunian object (TNO) of typical comet-size in that region. By monitoring thousands of stars at a cadence of about $10 \mathrm{~Hz}$, any TNO event will enable astrometry at sub-milli-arcsec level. The status of related researches is summarized.
\end{abstract}

\section{THE TYPICAL ANGULAR MEASUREMENTS IN OCCULTATION}

Occultation used to be the only tool for astronomers to do high angular resolution astronomy long before the development of interferometers. One is capable of doing better astrometry, resolving a close binary, measuring a stellar disk, locating planetary rings, studying a planetary atmosphere, and more. However, the configuration is not under control and, in general, not reproducible for most of them.

As a comparison, three different occulters is summarized below, namely, lunar occultation, an asteroidal event, and, a possible TNO event. The moon moves across the sky at a speed of $0.5 \mathrm{arcsec} / \mathrm{s}$. With high speed photometry at millisecond level, a milli-arcsec resolution of the background source can be reached. If observed at opposition, a main belt asteroid moves across the sky at a speed about $0.01 \mathrm{arcsec} / \mathrm{s}$. A sub-milli-arcsec resolution can be achieved with a high speed photometry faster than a tenth of a second. A TNO at 42 AU moves across the sky at a speed below 0.001 arcsec/s. A submilli-arcsec resolution is almost guaranteed. Moreover, with a high speed photometry at sub-millisecond level, such as the one used in the X-ray satellite, RXTE, an angular resolution below a micro-arcsec is possible provided that the background source is bright enough [1].

\section{OTHER FEATURES OF A TRANS-NEPTUNIAN OCCULTATION}

It is also appealing to compare a main belt event and a trans-Neptunian event other than their angular motion.

The typical duration of a main belt event is a few seconds since the ground speed of a shadow is about $10 \mathrm{~km} / \mathrm{s}$ (without considering the geodetic curvature) at opposition and the typical sizes of those known asteroids are roughly $10-100 \mathrm{~km}$. On the other hand, the Pluto has an angular diameter less than 0.1 arcsec. The ground speed of its shadow is slower than $25 \mathrm{~km} / \mathrm{s}$. All the observed TNOs have diameters larger than a few tens $\mathrm{km}$ (assuming an albedo of 0.04). That gives a duration similar to a typical main belt event.

One of the most interesting parts of the TNO occultation is its event rate. The event rate is basically proportional to the solid angle occupied by an object moving through the sky during a period of time. It is proportional to the size of an object times its angular displacement if observed away from its

\footnotetext{
ae-mail: skking@asiaa.sinica.edu.tw
}

This is an Open Access article distributed under the terms of the Creative Commons Attribution-Noncommercial License 3.0, which permits unrestricted use, distribution, and reproduction in any noncommercial medium, provided the original work is properly cited. 
stationary point. Thus, the event rate due to a main belt asteroid of kilometric size could be compatible with the event rate due to a TNO of a size about tens of kilometers as the latter is more than ten times farther away. As long as the size distributions of both populations are steep enough, which is, in fact, supported by the existing observations, the total solid angle, and hence, the event rate will be dominated by the faint-end objects of both the asteroids and the TNOs. Ivezić et al. [2] estimated that there might be 670,000 asteroids with a diameter larger than $1 \mathrm{~km}$ in the main belt. Based on several observations, Petit et al. [3] concluded that the number of TNOs with diameter larger than $100 \mathrm{~km}$ is on the order of a few $10^{4}$. That corresponds to a cumulative number of a few hundred TNOs, with diameters above a few tens kilometers, per square degree. Though, the survey of TNOs above the current threshold in a direct observation is not complete. Less than two thousand TNOs were catalogued so far. One may still argue that the total number of TNOs with a diameter above, say, 30 or 50 kilometers within the region of 30 to $50 \mathrm{AU}$ could be compatible with the total number of asteroids larger than one kilometer in the main belt. It means that the number of the main belt events and the number of trans-Neptunian events due to those big TNOs could be of the same order, which is about few per year ( $V_{\text {star }}$ brighter than 12$)$ [4] for a given site. By increasing the limiting magnitude, considering the TNO down to the typical size of a comet (a few $\mathrm{km}$ ), and the even larger space beyond the current Kuiper belt which is not accessible through a direct image, more occultation events should be expected.

The reflected sun light from a TNO is largely reduced according to an inverse fourth power law. Therefore, the foreground TNO will be virtually dark comparing to its background star. To distinguish a terrestrial exoplanet is still difficult. A nearby brown dwarf companion will be an easy target, though. As a TNO getting smaller or farther away, the diffraction effect could make an occultation invisible. At the same time, the angular size of a background star, might be compatible to its foreground TNO. A careful analysis is needed. The ephemeris of a known TNO has a larger uncertainty. For a comet-size TNO, a serendipitous occultation survey is the only possibility. The size of a shadow is roughly the same as the size of the occulter. Even though, a big TNO have a shadow of 100-1000 km, only. The shadow width of a comet-size TNO could be similar to the size of a small city. A serrendipitous event cannot be foreseen. The distance and other information of the occulter will have to be determined with the measurements of several other parameters. In order to measure its shape and moving direction, the use of a well-deployed array to get multi-chord data could be necessary. A multi-color observation would certainly help if diffraction effect is important.

The statistic properties of TNO occultation or any individual event is in itself an interesting problem. It could provide unique information of the whole population or the special properties of the foreground TNO. For instance, the size distribution of the small TNO is still unknown. The atmosphere of a giant TNO, such as those of Pluto or Eris, and its composition are interesting topic in planetary science. The shape, size and albedo of a TNO, especially of the small ones, is too far from us to be studied by other methods. All these features make TNO occultation a facinating and challenging problem.

\section{PERSPECTIVES}

Currently, a few hundred asteroidal events with fairly bright background stars are predicted and a certain fraction of them were observed every year. Most of the these observation were conducted by amateur networks using small telescopes. An observatory is still limited by the small number of events predicted. On the contrary, a portable facility has a high successful rate in such a campaign. Serendipitous surveys of trans-Neptunian event got no conceivable positive signal yet [5]. The original event rate estimate might be too optimistic. An orders of magnitude improvement shall be needed. Nevertheless, a promising future seems obvious as some on-going projects are expected to produce more high quality data. It is important to have accurate ephemeris of those solar system small bodies. It is also critical to have a more precise star catalog. Both will lead to better and more efficient prediction of occultation events. The space mission GAIA, the Pan-STARRS project, and, perhaps, the future LSST shall be able to provide those greatly improved data. On the other hand, the subsequent to the current 
Research, Science and Technology of Brown Dwarfs and Exoplanets

TAOS project [6] (namely, TAOS-2) with three 1-2 meter class telescopes, and, hopefully, more welldeployed arrays around the world, will be able to provide new insight on all those background objects at a very high angular resolution in the near future.

\section{References}

[1] Chang, H.-K. et al., MNRAS 378, (2007) 1287

[2] Ivezić, Ž. et al., AJ 122, (2001) 2749

[3] Petit, J.-M. et al., The Solar System Beyond Neptune (University of Arizona Press, Tucson 2008) 71

[4] Tanga, P. and Delbo, M., A\&A 474, (2007) 1015

[5] Zhang, Z.-W. et al., ApJ 685, (2008) L157

[6] Lehner, M. J. et al., PASP 121, (2009) 138 